\title{
Dynamic Handoff Priority Adjustment Based on Mobility-Awareness in Multimedia Cellular Networks
}

\author{
Chow-Sing Lin ${ }^{1}$, Pin-Jing Huang ${ }^{2}$ \\ ${ }^{1}$ Department of Computer Science and Information Engineering, National University of Tainan, Taiwan, Chinese Taipei \\ ${ }^{2}$ Department of Information Management, Southern Taiwan University, Tainan, Taiwan, Chinese Taipei \\ E-mail:mikelin@mail.nutn.edu.tw,Freda_huang@pegatroncorp.com \\ Received June 1, 2011; revised July 6, 2011; accepted July 15, 2011
}

\begin{abstract}
In multimedia cellular networks, when a Mobile Host requests multimedia services, it may experience handoffs to several cells before the request is completely served. If a target cell cannot provide adequate bandwidth for a service request, instead of directly dropping the request, the $M H$ is put into the handoff queue and hopefully the requested bandwidth can be satisfied by later released bandwidth. Obviously, it is important to properly assign priorities for queued handoff of $M H$ s based on their inborn dynamics to avoid unnecessary dropping. In this paper, we present a dynamic handoff priority adjustment scheme which applied a handoff queuing scheme to dynamically adjust handoff priority based on receiving signal strength, service class, and mobility of Mobile Hosts. In addition, idle bandwidth reserved by inactive $M H \mathrm{~s}$ is reallocated to urgent handoff $M H$ s to reduce the call dropping probability. The goal of the proposed dynamic handoff priority adjustment scheme is to further reduce call dropping probability while still maintaining high bandwidth utilization and acceptable call blocking probability on multimedia cellular networks.
\end{abstract}

Keywords: Multimedia Cellular Network, Handoff Priority, Handoff Queue, Quality of Service, Mobility

\section{Introduction}

In recent years, due to rapid advances in networking technology, providing multimedia service on cellular network now becomes feasible. The types of accessible multimedia contents, such as data, voice, music, and video have grown explosively [1-4]. On multimedia cellular networks, Mobile Hosts ( $M H \mathrm{~s})$ may access various types of services, and each requires different Quality-of-Service (QoS). During the service time, a $M H$ may generally move across several cells. Due to the unpredictable mobility of $M H \mathrm{~s}$, how to maintain a consistent QoS becomes more difficult and challenging on multimedia cellular networks.

Three metrics are generally used for measuring QoS: Call Blocking Probability, Call Dropping Probability, and Bandwidth Utilization. For a new call, if the origin cell cannot provide sufficient bandwidth, it will be blocked [5-6]. The call blocking probability (CBP) denotes the probability for a new call to be denied by origin cell. On the other hand, for an on-going call, if its origin cell no longer has enough bandwidth to maintain the requested service, it issues a handoff request to hand over the service to the target cell. However, the on-going call is dropped if the target cell also has insufficient bandwidth for continually providing service. The probability of rejecting a handoff request due to the insufficient bandwidth is called call dropping probability (CDP). From a client's perspective, it is more intolerable to drop an on-going service, than to block a service that has yet to be established [3]. Therefore, with limited bandwidth in a cell, satisfying handoff requests of on-going calls is more important and thus gives high priority than new calls, i.e., the CDP should be kept as low as possible in a cell [7-8]. Finally, the bandwidth utilization (BU) denotes the average bandwidth utilization of cells in a multimedia cellular network.

The typical infrastructure of cellular network is made of several hexagons called cells, and $M H \mathrm{~s}$ in a cell are served by a base station (BS). Between two cells there is an overlap area, called handoff area or handoff zone. $M H \mathrm{~s}$ in this handoff area can receive signals from both BS. When the Received Signal Strength (RSS) of origin cell is below a certain threshold, a $M H$ is handed over to the target cell to continue the service. To avoid disrupting connections during a handoff process, a seamless 
cellular network is constructed [6,9]. MHs can move from one cell to another by soft handoff to keep enjoying the service without disruption [11]. In order to increase system efficiency and therefore to serve more users in cellular networks, nowadays, cells in a cellular network tends to shrink from macro cells to micro/pico cells [11, 12]. Nevertheless, such an approach inevitably increases the number of handoffs among cells, leading to an increase of the CDP [13-15]. Figure 1 depicts the relationship of $R S S$ and handoff process of $M H \mathrm{~s}$. When $M H_{a}$ moves toward the target cell, $\mathrm{Cell}_{B}$, its $R S S$ from $B S_{A}$ changes accordingly. When $M H_{a}$ reaches its handoff threshold of the $R S S$ equal to $A$ ', it issues a handoff request to the BS of target cell, $B S_{B}$. The $R S S$ of $M H_{a}$ from $\mathrm{Cell}_{A}$ gets weaker while $M H_{a}$ moving toward the $\mathrm{Cell}_{B}$. Once it reaches the received threshold of $B$, the $R S S$ from $B S_{A}$ will be too weak to establish a workable connection to $M H_{a}$. At this point, the connection of $M H_{a}$ will be disrupted if $B S_{B}$ is unable to provide sufficient bandwidth for handoff $[16,17]$. In other words, the handoff to $\mathrm{Cell}_{B}$ for $M H_{a}$ is issued at time $t_{1}$ and must be completed before the time $t_{2}$ to avoid service interruption.

Generally, once a $M H$ enters a handoff zone, it issues a handoff request to the target cell. If the target cell cannot provide adequate bandwidth for on-going services either by bandwidth reservation scheme [18] or by guard channel scheme [19], instead of directly dropping the request, the $M H$ is put into the handoff queue, and hopefully the requested bandwidth can be satisfied later by released bandwidth. Obviously, properly assigning priorities based on their inborn dynamics to avoid unnecessary dropping is important for queued handoffing $M H$ s. In multimedia cellular networks, $\mathrm{MHs}$ have various characteristics such as requested service class, moving velocity, mobility, and RSS, etc., which can be used to

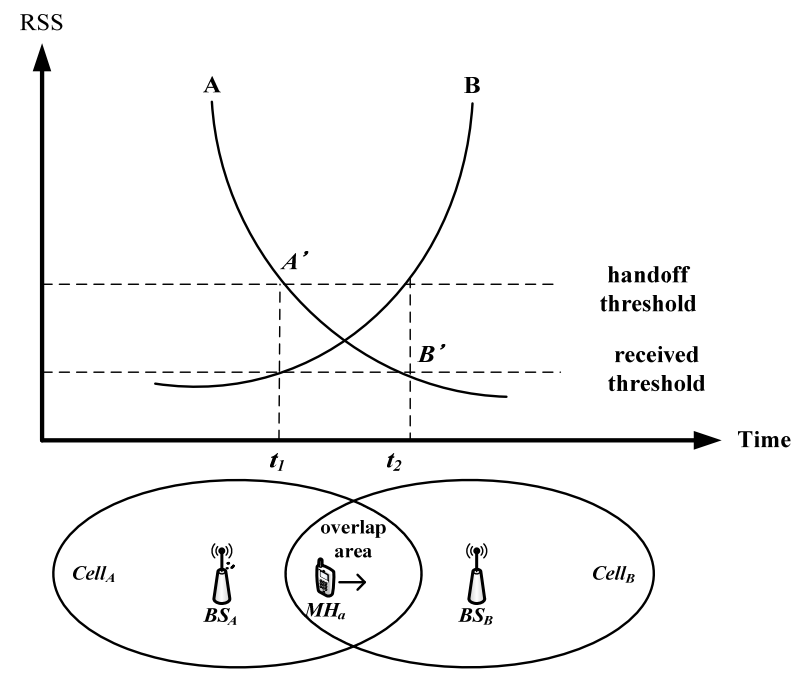

Figure 1. Handoff and RSS [16]. determined the handoff priority of an on-going call. Particularly, the mobility of a $M H$ may change erratically, and therefore, its priority in handoff queue must be dynamically adjusted based on its mobility to reflect the urgency of handoff. For example, if a $M H$ in handoff queue stops moving (stands still) or even moving away from the target cell, its handoff priority should be gradually decreased. On the other hand, the handoff priority of a $M H$ in handoff queue should be timely raised if the $M H$ accelerates toward the target cell.

In this paper, we proposed a dynamic handoff priority adjustment (DHAP) scheme to order queued handoffing requests of $M H$ s. To specify whether a $M H$ is continuously moving or rarely moving, the mobility of a $M H$ was classified into active mode and inactive mode based on a threshold of the moving speed. In addition, the DHAP consisted of bi-handoff queues, which were $a c$ tive handoff queue and inactive handoff queue. Handoffing $M H \mathrm{~s}$ in active and inactive mode failing to acquire adequate bandwidth in target cell were inserted into active handoff queue and inactive handoff queue, respectively. The priority of a $M H$ in active handoff queue was periodically adjusted by its dwelling time which indicates how soon the $M H$ is to be handoffed to the target cell. On the other hand, the priority of a $M H$ in inactive handoff queue is not updated or updated at a large time interval since the $M H$ rarely moves. The migration between active and inactive handoff queues might happen when the mobility of a queued handoff $M H$ changes. Besides, we also classify $M H$ s who successfully reserve bandwidth in target cells into mobile set and immobile set based on their mobility. Idle bandwidth reserved by inactive $M H \mathrm{~s}$ in immobile set is reallocated to urgent handoff $M H$ s to further reduce the CDP.

The remainder of this paper is organized as follows. In Section 2, related works of handoff queuing schemes are investigated. The proposed DHPA scheme is presented in Section 3. Section 4 presents simulation results and analyses. Finally, concluding remarks are given in Section 5.

\section{Related Works}

To reduce the probability of terminating handoff calls due to the shortage of available bandwidth in target cell, handoff queuing scheme have been widely studied in recent years. The priority of a handoff call in a handoff queue can be determined by single or multiple factors, which are described as follows.

The simplest strategy of determining priority for a queued $M H$ is based on its order of entering handoff area, such as First-In-First-Out (FIFO). A handoff call is added into FIFO queue if its target cell has no sufficient avail- 
able bandwidth to satisfy the service. When there is bandwidth released in a cell, the $M H$ in the handoff queue with the earliest handoff time will obtain the bandwidth to process its handoff. Obviously, after entering a handoff area, a $M H$ may stop moving, or even move backward. Without considering other factors, FIFO scheme cannot truly reflect the urgency of handoff due to the erratic mobility of $M H \mathrm{~s}$. Qian and Feng [20] present the minimum-dwelling-time scheme to prioritize handoff requests. The priority of handoff requests is determined by its estimated dwelling time in the handoff area. A $M H$ with shorter dwelling time has higher probability to handoff to its target cell, and hence should have the higher handoff priority. A measurement-based prioritizing scheme (MBPS) [21] has been developed for prioritizing the queue instead of FIFO scheme. In MBPS, the priority is determined only based on the RSS of a $M H$. Once a $M H$ enters the handoff area, its $R S S$ may continuously vary during the handoffing time. The stronger the RSS of a $M H$, the lesser urgency of handoff, and therefore the lower priority in the handoff queue. Compared with the FIFO scheme, the MBPS using the RSS to determine the handoff priority is more reasonable but still not accurate for reflecting the urgency of handoff calls.

To more precisely reflect a $M H$ 's status, instead of using only one single factor, several studies apply multiple factors for fine-tuning the handoff priority. Ebersman and Tonguz [22] proposed a signal prediction priority queuing (SPPQ) method using not only RSS but also moving speed, which is interpreted as the change of $R S S$ ( $\triangle R S S$ ) to determine the priority of a $M H$. With the same time interval, a higher value of $\triangle R S S$ means faster moving speed of a $M H$. Since a $M H$ with high moving speed is more likely to be handoffed to its target cell than one with low moving speed, it should have a higher priority in handoff queue. Therefore, compared with queuing schemes considering only one factor, the SPPQ assigns a more proper handoff priority to an on-going $\mathrm{MH}$. However, in SPPQ only one type of service is considered. Chang and Leu [16] further extended the SPPQ algorithm, called Signal Strength for Multimedia Communications (SSMC) to handle multiple service types of multimedia traffic. In SSMC, the service class $\left(C_{i}\right), R S S_{i}$ and $\triangle R S S_{i}$ are considered to determine the priority of a $M H_{i}$ in handoff queue, $P_{i}$, which can be estimated by (1).

$$
P_{i}=C_{i} \times \Delta R S S_{i} \frac{1}{\Delta R S S_{i}}
$$

The SSMC scheme fails to consider the situation when a $M H$ stops moving or is even moving backward. For example, when a $M H$ close to the boundary of handoff area becomes temporary immobile, as shown in (1), its handoff priority $P_{i}$ suddenly becomes zero as the value of $\triangle R S S$ equals zero. If the $M H$ reinstates mobility, its handoff priority may be too low to acquire available bandwidth in time, leading to an increase of call dropping probability.

In this paper, we propose a Dynamic Handoff Priority Adjustment (DHPA) scheme to further extend the SSMC scheme. In DHPA, we similarly use RSS, $\triangle R S S$, and service class to determine the priority of a queued $M H$ for handoff. In addition, the DHPA scheme further monitors the mobility of queued $M H \mathrm{~s}$ handoff to dynamically and gradually adjust handoff priorities based on their dwelling time. Idle bandwidth reserved by immobile $M H \mathrm{~s}$ is also utilized to further reduce the CDP.

\section{Dynamic Handoff Priority Adjustment}

In this paper, for simplicity of analyses, a precise moving prediction algorithm $[9,23]$ is assumed so that a handoffing $M H$ issues bandwidth reservation request to the target cell once it enters the handoff area. However, the DHPA could be easily extended to multiple target cell approach with reserving bandwidth on target cells based on their handoff probability [18]. In general, a MH may move in various directions. Then, we assumed a $M H$ moving toward the target cell is called moving forward; a $M H$ moving away from the target cell is called moving backward. In DHPA, depending on $\triangle R S S$, the mobility of a $M H$ was classified as active mode or inactive mode. Accordingly, we also denoted bi-handoff queues in a cell. One was called active handoff queue, and the other was called inactive handoff queue. A handoffing $M H$ failing to acquire bandwidth from a target cell was inserted into the active or inactive handoff queue based on its mobility. The handoff priorities of the queued handoff $M H \mathrm{~s}$ were periodically adjusted to timely reflect the change of their mobility so that an urgent $M H$ could have a higher priority to acquire released bandwidth in target cell to avoid service interruption. Basically, when there was bandwidth released in the target cell, $M H \mathrm{~s}$ in the active handoff queue were served first, followed by those in the inactive handoff queue if any bandwidth left. MHs may migrate between active handoff queue and inactive handoff queue when their mobility changes with time. Figure 2 shows the handoff queuing scheme.

\subsection{The Classification of the Mobility of MHs}

In this paper, the $\triangle R S S$ of $M H_{i}$ at time $t$ was primarily determined by $\triangle R S S$,

$$
\Delta R S S_{i}^{t}=\frac{R S S_{i}^{t}-R S S_{i}^{t-1}}{\Delta T}
$$




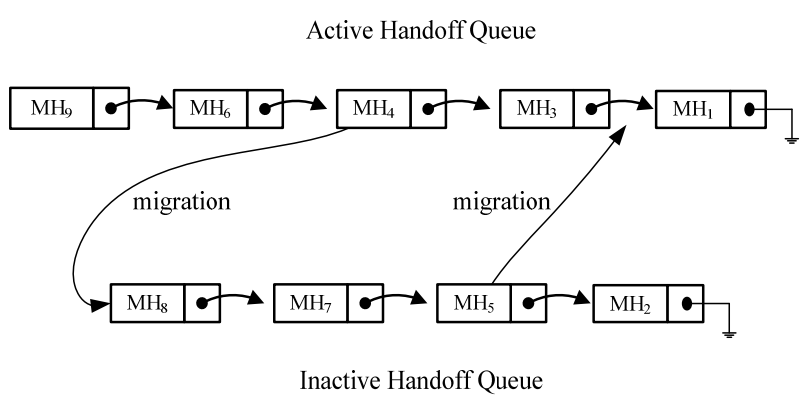

Figure 2. Handoff queuing scheme.

where $R S S_{i}^{t}$ and $R S S_{i}^{t-1}$ denoted the $R S S$ of $M H_{i}$ at time $t$ and time $t-1$, respectively, and $\Delta T$ equaled the difference between two consecutive time $t$ and $t-1$. If the $\triangle R S S$ was positive, it was moving backward to origin cell; if negative, it was moving toward target cell; if the $\triangle R S S$ equal to zero, it was immobile. The mobility of a $M H$ was classified into active and inactive modes, which can be determined by,

Mobility $=\left\{\begin{array}{l}\text { active, if }\left|\Delta R S S_{i}^{t}\right| \times w_{1}+\left|\Delta R S S_{i}^{t-1}\right| \times w_{2} \geq \delta \\ \text { inactive, if }\left|\Delta R S S_{i}^{t}\right| \times w_{1}+\left|\Delta R S S_{i}^{t-1}\right| \times w_{2}<\delta\end{array}\right.$,

where $\delta$ denoted the threshold of mobility, and $w_{1}$ and $w_{2}$ represented the weights of two consecutive $\triangle R S S$. If the value of $\left|\Delta R S S_{i}^{t}\right| \times w_{1}+\left|\Delta R S S_{i}^{t-1}\right| \times w_{2}$ of a $M H$ was larger than or equal to $\delta$, it means that the $M H$ kept moving at reasonable speed. Its mobility was in active mode. In contrast, if the value of $\left|\Delta R S S_{i}^{t}\right| \times w_{1}+\left|\Delta R S S_{i}^{t-1}\right| \times w_{2}$ of a $M H$ was less than $\delta$, it means that the $M H$ rarely moved or even stopped moving, and could be classified as in inactive mode. In addition, when a $M H$ moved out of a handoff area, the handoff request was no longer valid and therefore the $M H$ must be moved out of the handoff queue.

\subsection{Call Admission Control}

In order to avoid disruption of on-going services, the target cell provides the released bandwidth to on-going connections prior to new calls. Consequently, the DHPA provides different call admission control to new calls and handoff calls. When a cell, $C_{i}$, received a request from a new call $M H_{i}$, it first determined whether there was sufficient available bandwidth $\left(B W^{a}\right)$ to accept the requested bandwidth $\left(B W_{i}^{r}\right)$ of the new call. If the available bandwidth was sufficient, the cell accepted the request, and this new call became an on-going call. On the other hand, if the cell had no sufficient bandwidth, the new call was inserted into a new call queue waiting for available bandwidth. Figure 3 shows the flow chart of call admission control for new calls.

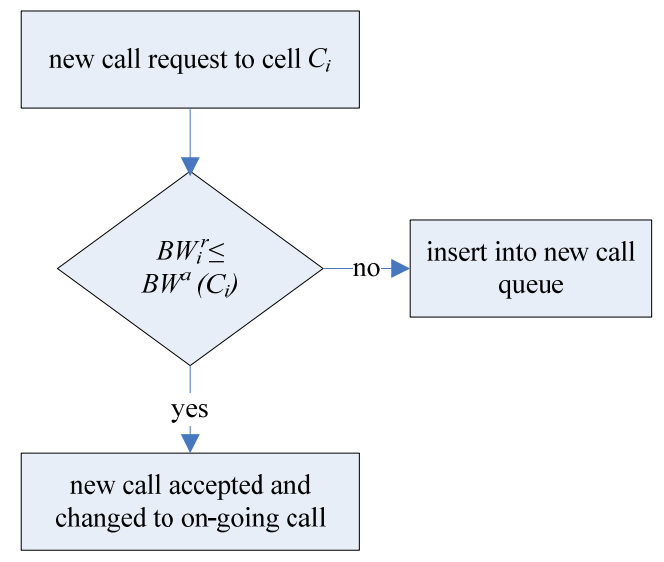

Figure 3. Call admission control for new call.

When an on-going call entered a handoff zone at time $t$, i.e., the current $R S S_{i}^{t}$ of $M H_{i}$ was less than the pre-defined signal threshold of issuing handoff, $R S S_{i}^{\text {issuehandoff }}$, it issued a handoff request to the target cell. Its mobility was changed to active, and then the $M H$ tried to reserve available bandwidth in the target cell to avoid service interruption. If the required bandwidth was less than the available bandwidth of the target cell, the target cell granted the bandwidth reservation request. The $M H$ was then added to the mobile set, which is defined in the later section of bandwidth reservation. If the target cell had insufficient available bandwidth for the handoff request, the target cell denied the bandwidth reservation request, and the handoff request was then put into the handoff queue with the initial priority calculated by the DHPA algorithm. Figure 4 shows the flow chart of call admission control for handoff calls.

\subsection{Bandwidth Reservation}

Generally, the mobility of a $M H$ might change after successfully reserving bandwidth. The DHPA algorithm classified those handoffing $\mathrm{MHs}$ into two sets, which are the mobile set and the immobile set. The mobile set $\left(U_{m}\right)$ is a set of $M H \mathrm{~s}$ with the mobility in active mode; the immobile set $\left(U_{i m}\right)$ is a set of $M H$ s with the mobility in inactive mode. A $M H$ with successful bandwidth reservation is initially put into the mobile set. Furthermore, a $M H$ may migrate between mobile set and immobile set based on its mobility which might be changed with time. When a $M H$ successfully reserved bandwidth in a target cell, DHPA would initially put those $M H \mathrm{~s}$ in the mobile set. These $M H \mathrm{~s}$ are monitored periodically to check if a handoff is required by examining the strength of its received signal. If the $R S S^{t}$ was less than the $R S S^{\text {handoff }}$, the $M H$ was handoffed to the target cell. In DHPA, before handoffed to the target cell, the mobility of $M H \mathrm{~s}$ was continuously monitored and updated. If the $M H$ was still 


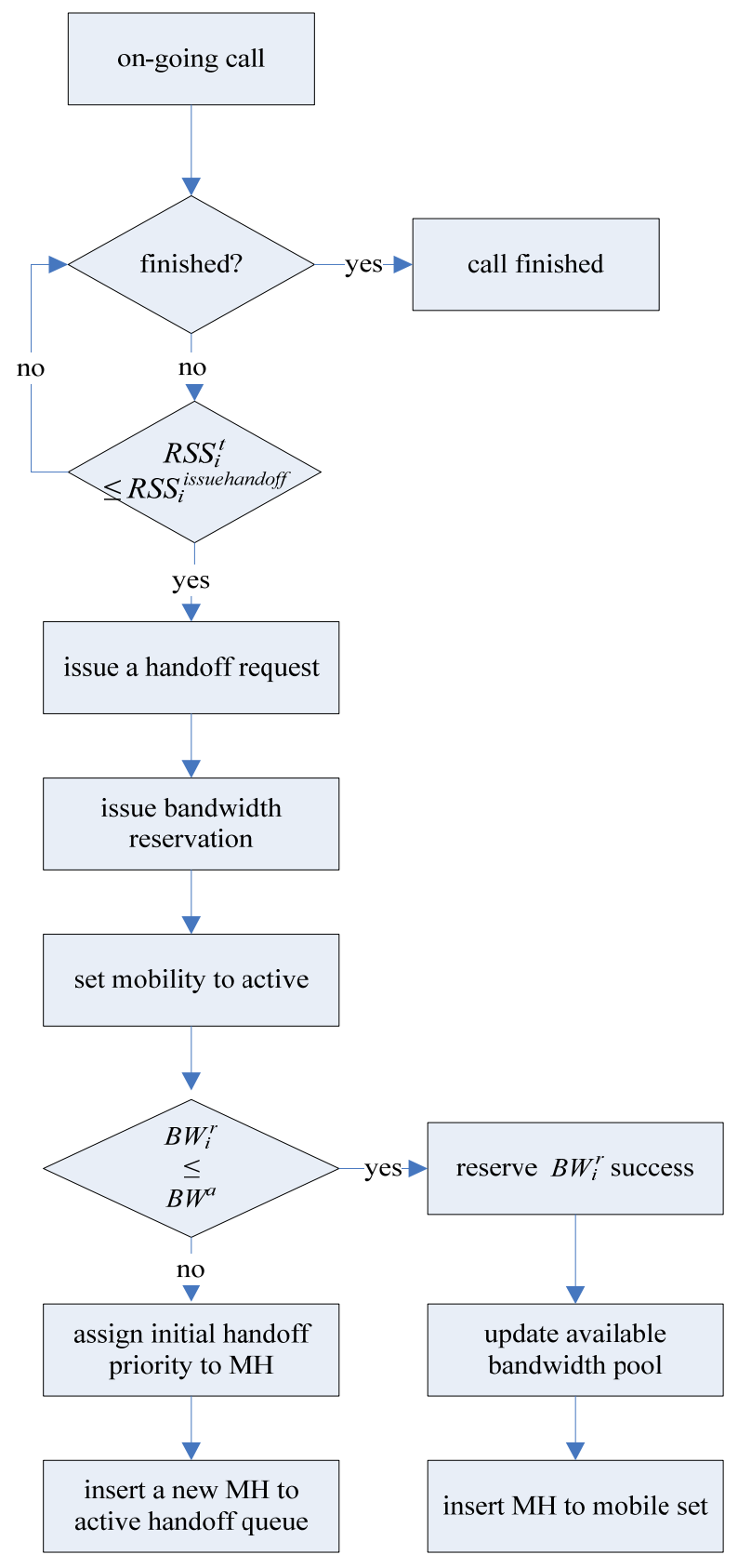

Figure 4. Call admission control for handoff call.

in active mode, it stays in mobile set; otherwise, it migrates to the immobile set. Figure 5 shows the process of periodically updating $\mathrm{MHs}$ in mobile set.

On the other hand, when a $M H$ successfully reserving bandwidth in the target cell changed to inactive, it was added into immobile set, $U_{i m}$. A $M H$ in $U_{i m}$ might be asked to release the reserved bandwidth in order to avoid dropping handoff calls, because its reserved bandwidth in the target cell might not be used for a while. In this paper, the bandwidth of inactive $M H \mathrm{~s}$ was aggregated as a reserved bandwidth pool (RBpool), which was used to

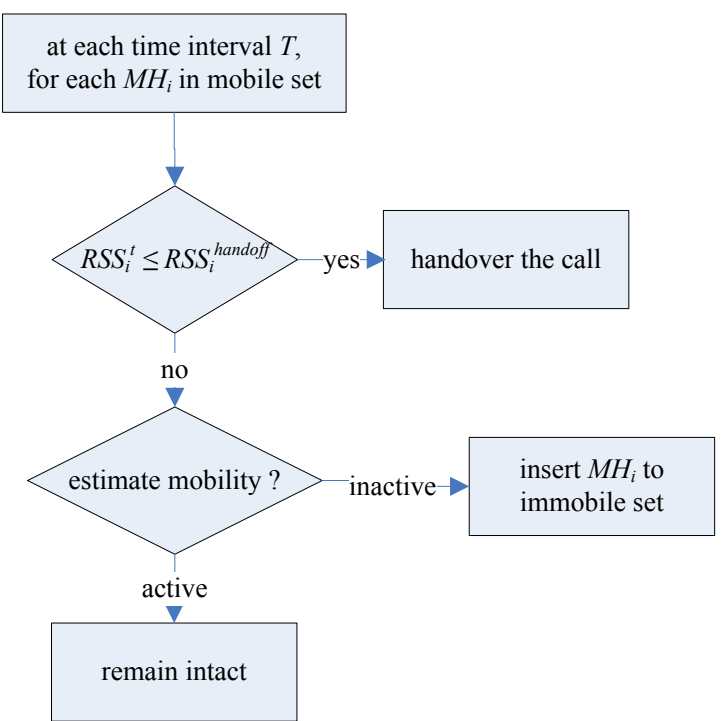

Figure 5. Process of periodically updating $M H$ s in mobile set.

serve those $M H$ s that require urgent handoffs but had failed to reserve bandwidth previously. Again, before handoffed to the target cell, the mobility of $M H \mathrm{~s}$ was continuously monitored and updated.

If a $M H$ became mobile, before it changed to active mode, we checked whether there was enough bandwidth in RBpool to be allocated to the $M H$. If so, the requested bandwidth in RBpool was allocated to the $M H$; otherwise, the $M H$ was inserted into the inactive handoff queue. Finally, the mobility of the $M H$ was changed to active. Figure 6 shows the process of periodically updating $M H \mathrm{~s}$ in immobile set in immobile set.

\subsection{Handoff Priority Adjustment}

Recall that when a $M H$ entered the handoff area, it issued a handoff request to the target cell. If the target cell could not provide adequate bandwidth for requested service, the $M H$ was inserted into the handoff queue, and hopefully the requested bandwidth could be satisfied by later released bandwidth. Before inserting the $M H_{i}$ into the handoff queue, the initial priority, $P_{i}^{\text {init }}$, was computed by (4), where $S C_{i}, \Delta R S S_{i}$, and $R S S_{i}$ denoted the priority of requested service class, the change in received signal strength, and received signal strength of $M H_{i}$, respectively.

$$
P_{i}^{\text {init }}=S C_{i} \times\left|\Delta R S S_{i}\right| \times \frac{1}{R S S_{i}}
$$

A higher class of services had a higher value of $S C$ and should be served first. $\triangle R S S$ is the change of signal strength between two moves. The faster a $M H$ moved, the larger the $\triangle R S S$ and the higher handoff priority. 


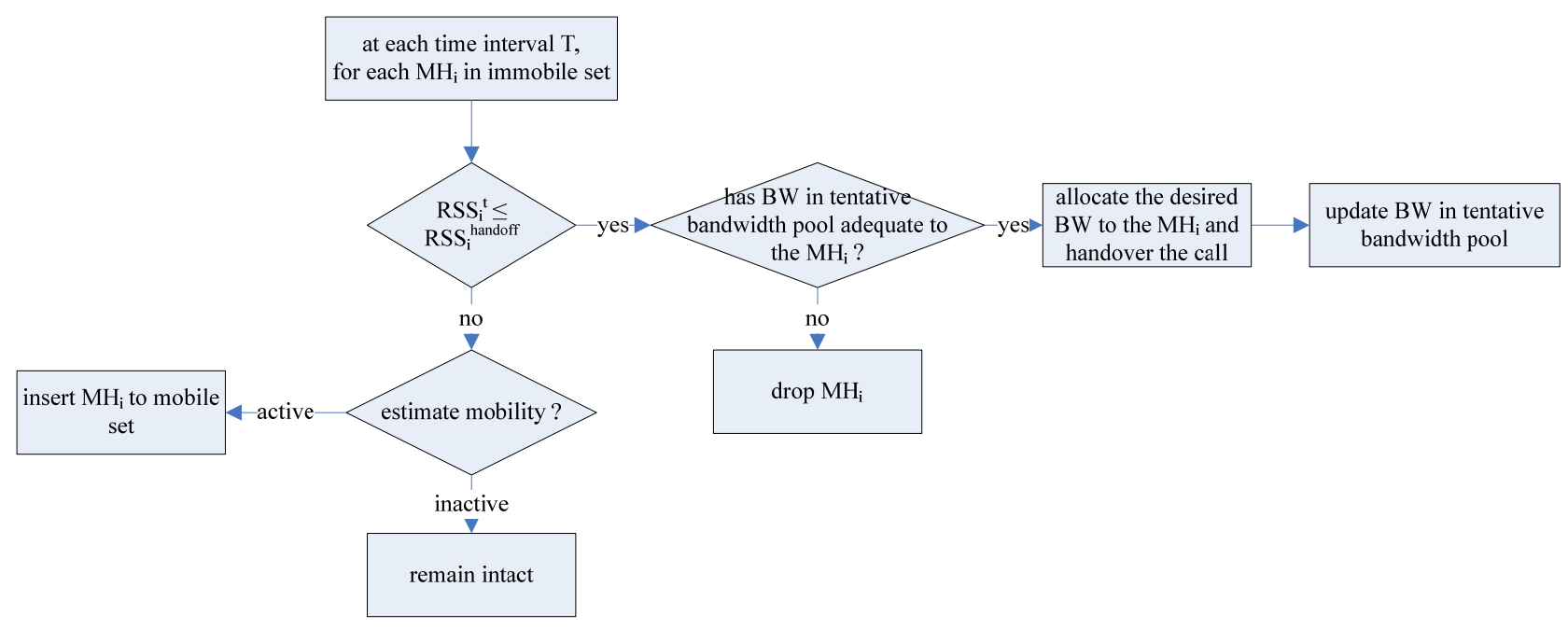

Figure 6. Process of periodically updating $M H s$ in immobile set.

When a $M H$ moved further away from its origin cell, the signal receiving from the origin cell became weaker, resulting in a larger $1 / R S S$ and hence leading to an increase of the handoff priority.

For those $M H$ s residing in the active handoff queue, at each time interval, first we checked if a $M H$ was moving out of the handoff area. If a $M H$ in active mode kept moving toward the target cell and was ready to handoff, i.e., $R S S_{i}^{t}<=R S S_{i}^{\text {handoff }}$, the $M H$ was handoffed to the target cell if there was enough bandwidth in the target cell or in the RBpool; otherwise, the $M H$ was dropped. On the other hand, if a $M H$ moved backward to the origin cell, i.e., $R S S_{i}^{t}>=R S S_{i}^{\text {handoff }}$, the $M H$ was removed from the active handoff queue. If a $M H$ still stayed in the handoff area, we next determined its mobility. If it changed to inactive, it was migrated to the inactive handoff queue; otherwise, its priority in the active handoff queue should be periodically updated. In this paper, the DHPA periodically updated handoff priority of a handoffing $M H$ according to its dwelling time, which indicated how soon a $M H$ was expected to be handoffed to the target cell. Intuitively, a $M H$ which had shorter dwelling time should have higher probability to be handoffed into the target cell, and thus should be given a higher handoff priority. Assume $D T_{i}^{t}$ denoted the dwelling time of $M H_{i}$ in the handoff area, $R S S_{i}^{\text {handoff }}$ denoted the threshold of receive signal strength to handoff, $w_{1}$ and $w_{2}$ denoted the relative weights of $R S S_{i}^{t}$ and $R S S_{i}^{t-1}$, then the dwelling time of $M H_{i}$ in the active handoff queue could be estimated as (5),

$$
D T_{i}^{t}=\frac{R S S_{i}^{t}-R S S_{i}^{\text {handoff }}}{\left|\Delta R S S_{i}^{t}\right| \times w_{1}+\left|\Delta R S S_{i}^{t-1}\right| \times w_{2}} .
$$

Based on the dwelling time of $M H_{i}$, we used (6) to periodically update its handoff priority, $P_{i}^{t}$,

$$
P_{i}^{t}= \begin{cases}P_{i}^{t-1} \times\left(1+\frac{1}{D T_{i}^{t}}\right), & D T_{i}^{t} \neq 0 \\ P_{i}^{t-1}, & D T_{i}^{t}=0\end{cases}
$$

where $P_{i}^{t}$ and $P_{i}^{t-1}$ denoted the handoff priority for $M H_{i}$ at time $t-1$ and $t$, and $D T_{i}^{t}$ indicated the dwelling time at time $t$. Figures $\mathbf{7}$ and $\mathbf{8}$ show the process and pseudo code of updating $\mathrm{MHs}$ ' statuses in active handoff queue.

On the other hand, if $M H \mathrm{~s}$ rarely moved in the handoff area, they became inactive and thus were inserted into inactive handoff queue. The priority of a $M H$ for inserting into the inactive queue, $P_{i}^{\text {inactive }}$, could be calculated by (7)

$$
\begin{aligned}
& P_{i}^{\text {inactive }} \\
& =S C_{i} \times \frac{1}{R S S_{i}^{t}} \times\left(R S S_{i}^{\text {issuehandoff }}-R S S_{i}^{t}\right) \\
& =S C_{i} \times \frac{R S S_{i}^{\text {issuehandoff }}}{R S S_{i}^{t}}-1
\end{aligned}
$$

Because the RSS of a $M H$ in the inactive queue was seldom changed, its priority would not to be changed periodically, or could be updated at a large time interval.

However, similar to $M H \mathrm{~s}$ in active handoff queue, they had to periodically check their residence and mobility in handoff area. If a $M H$ in inactive mode was ready to be handoffed, i.e., $R S S_{i}^{t}<=R S S_{i}^{\text {handoff }}$, the $M H$ was handoffed to the target cell if there was enough bandwidth in the target cell or in the RBpool for this handoff; otherwise, the $M H$ was dropped. On the other hand, if a $M H$ gradually moved backward to the origin cell, i.e., $R S S_{i}^{t}>=R S S_{i}^{\text {handoff }}$, the $M H$ was removed from the inactive handoff queue. Furthermore, if a $M H$ still stayed in the handoff area, we next estimated its mobility. If the mobility of a $M H$ changed to active, it would be mi- 


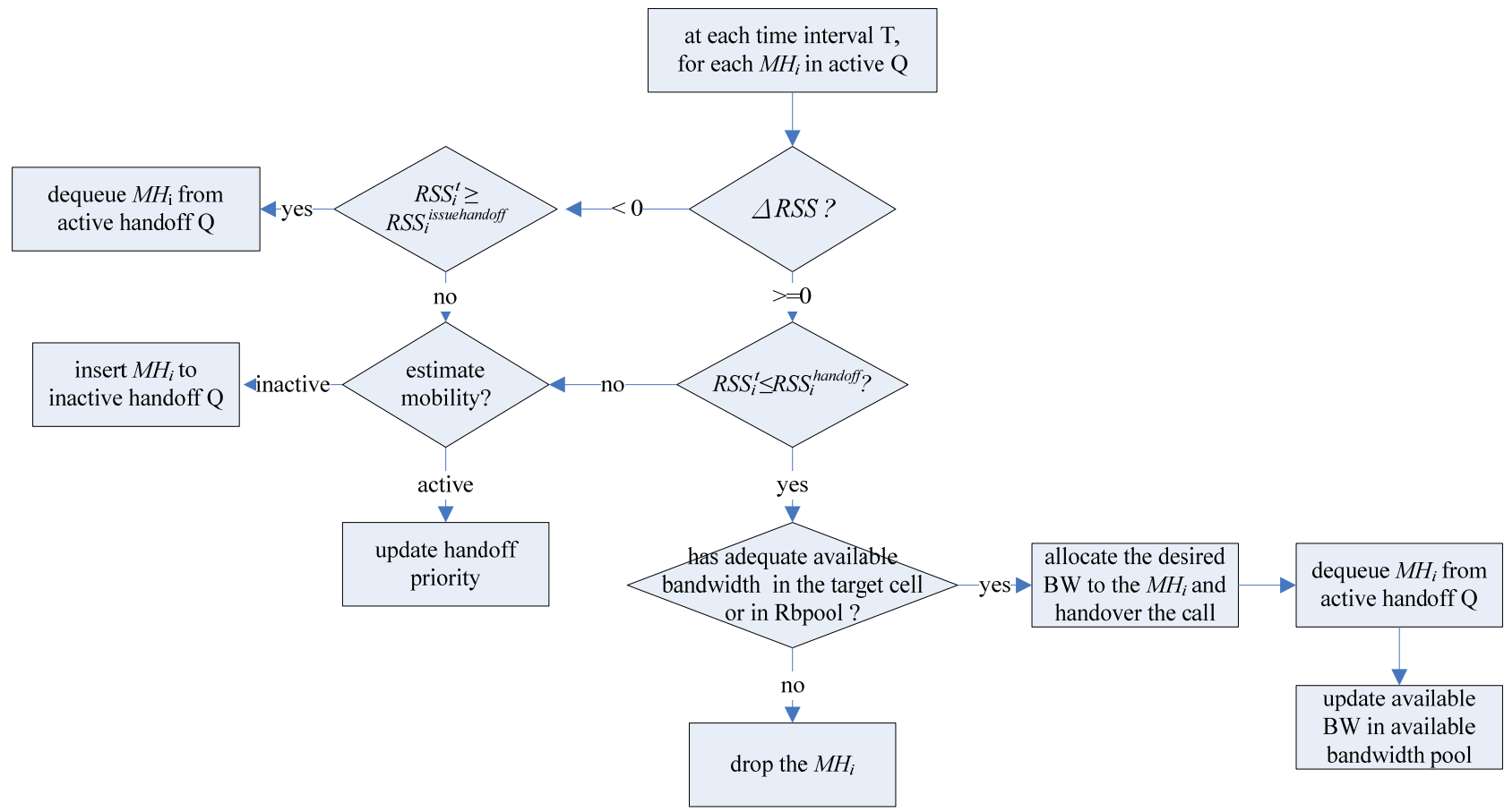

Figure 7. Process of updating MHs' statuses in active handoff queue.

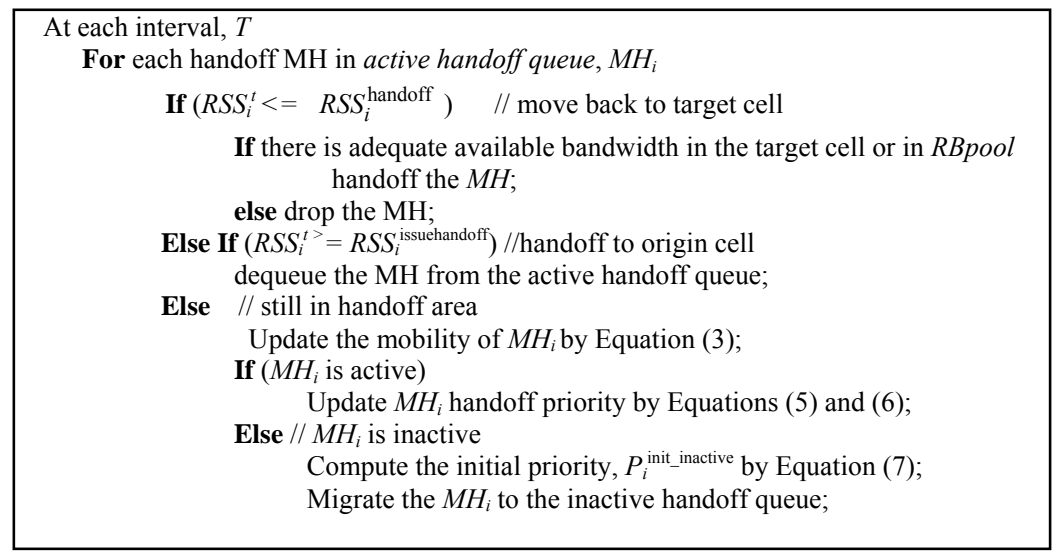

Figure 8. Pseudo code of updating $M H s$ in active handoff queue.

grated to the active handoff queue with the initial priority calculated by (4); otherwise it remained intact. Figures 9 and $\mathbf{1 0}$ show the process and pseudo code of updating $M H$ s' statuses in inactive handoff queue.

\subsection{Reallocation of Released Bandwidth}

Once a target cell finished providing the service for a $M H \mathrm{l}$, the released bandwidth was returned back to the available bandwidth pool for reallocation. If there were any $\mathrm{MHs}$ in the handoff queues and the new call queue, the latest available bandwidth was allocated to queues in order of priority, which was active handoff queue first, then inactive handoff queue, and finally the new call queue. First, if there were pending $M H$ s in active handoff queue, we searched for the $M H$ with the highest priority and its requested bandwidth was less or equal to the available bandwidth. If we found one, its requested bandwidth was reserved, and it was removed from active handoff queue and joined the mobile set. The available bandwidth was then updated accordingly. Such a process was repeated until no $M H$ satisfied the above criteria could be found. If there was any available bandwidth left, the above process was again applied to $\mathrm{MHs}$ in the inactive queue. After applying the above two steps, if there was available bandwidth left, it was allocated to pending new calls if there was any. The process of reallocation of released bandwidth detailed process flow is shown in 


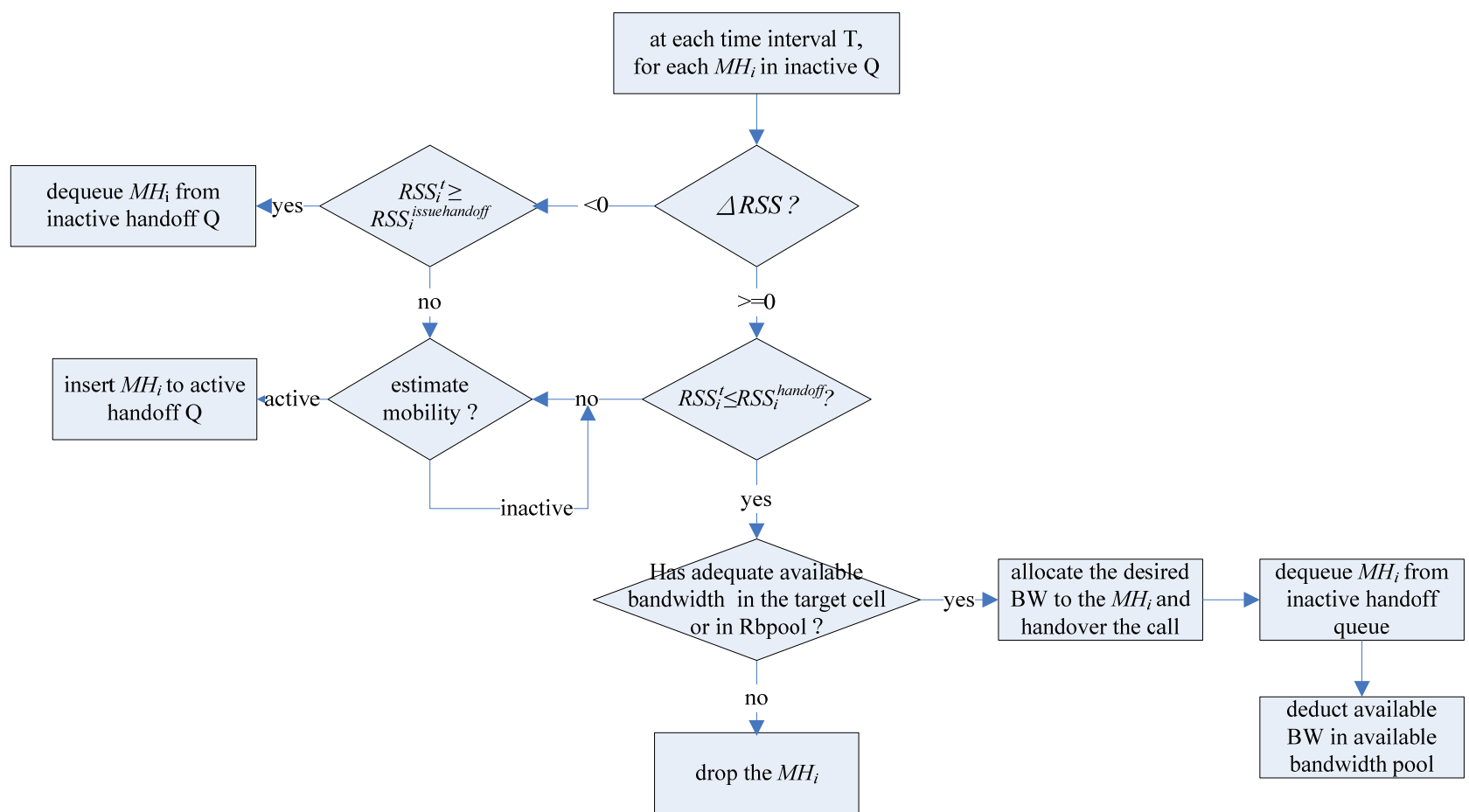

Figure 9. The process of updating $M H s^{\prime}$ statuses in inactive handoff queue.

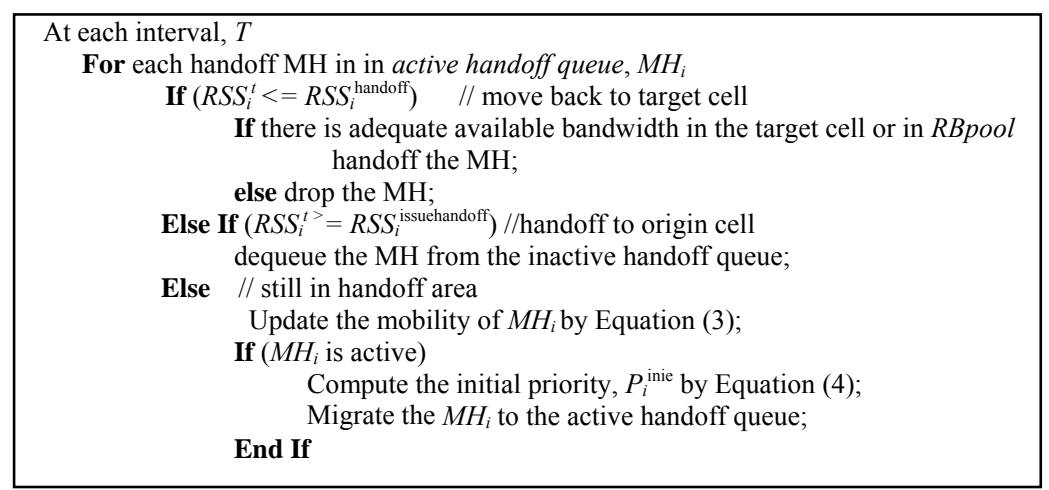

Figure 10. Pseudo code of updating $\mathbf{M H s}$ ' statuses in inactive handoff queue.

\section{Figure 11.}

\subsection{The State Transition of MHs in Handoff Area}

Figure 12 shows the state transition of a $M H$ in handoff area. In general, once a $M H$ entered the handoff area, it was in the "Enter Handoff Area" state and a request of bandwidth reservation in the target cell was issued. If the bandwidth reservation was granted, the $M H$ was added into the mobile set and changed to "Mobile Set" state; otherwise, the $M H$ was inserted into the active handoff queue and changed to "Active queue" state. $M H \mathrm{~s}$ in "Mobile Set"/“Immobile Set" state might change to "Immobile Set"/"Mobile Set" state if their mobility be came inactive/active. Similarly, $M H$ s in "Active Queue"/ "Inactive Queue" state might change to "Inactive Queue"/ "Active Queue" state if their mobility became inactive/ active. A $M H$ in "Immobile Set" state might change to "Inactive Queue" state if its reserved bandwidth in RBpool was allocated to other $M H$ s for saving call dropping. Finally, when a $M H$ in "Inactive Queue" was allocated the requested bandwidth in the target cell, it changed to the "Immobile Set" state and its reserved bandwidth was then added to the RBpool.

\section{Simulation Results}

The simulation model was assumed to be on a multimedia cellular network, which consisted of 25 cells. The 


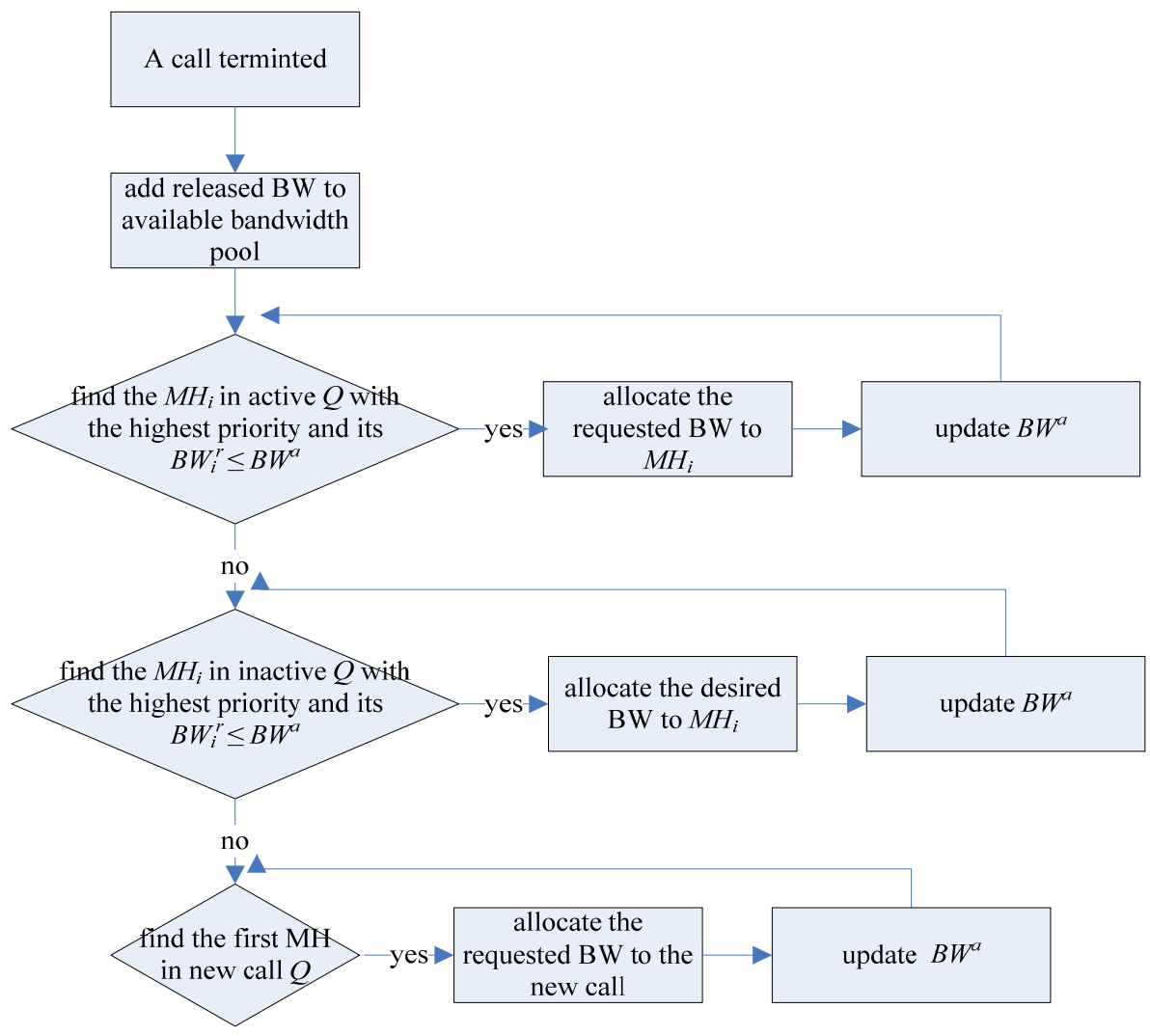

Figure 11. Process of reallocation of released bandwidth.

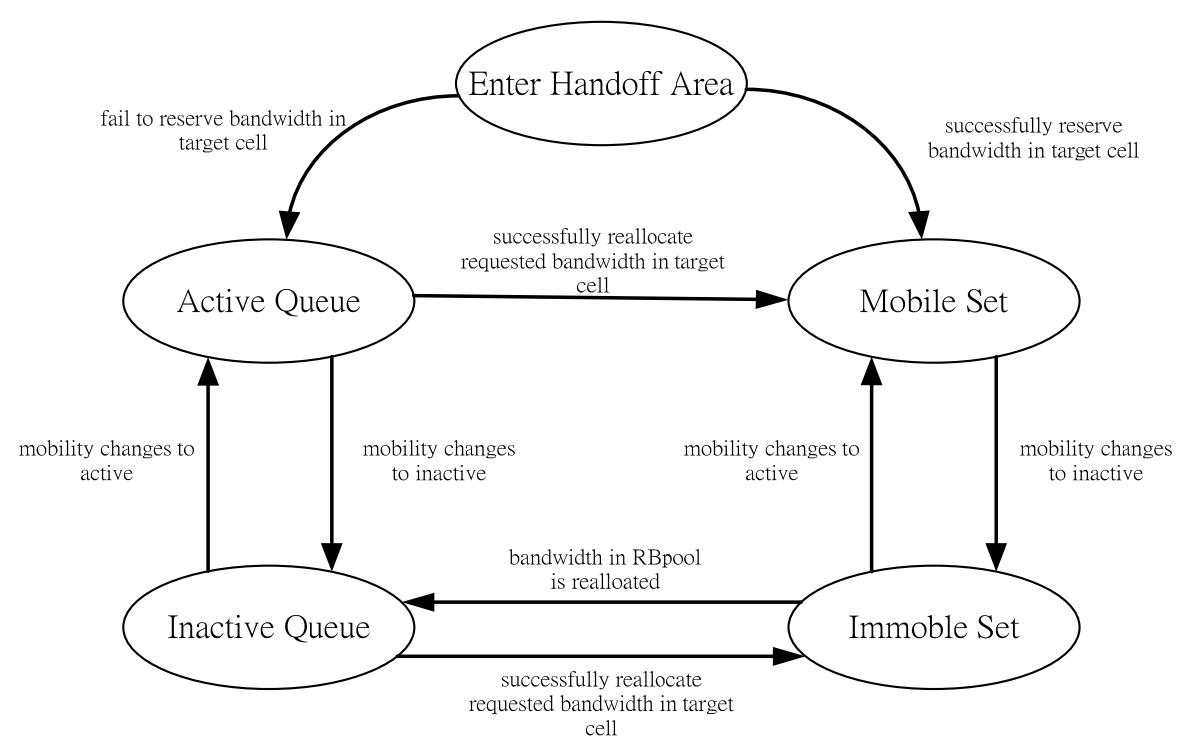

Figure 12. State transition of a $M H$ in handoff area.

coverage of a cell was $4 * 4 \mathrm{~km}^{2}$. Each cell had 50 channels, and each channel had the network capacity of 64 kbps. There were three service classes in the simulation listed as in Table 1 [16]. The call arrival rate followed Poisson distribution, and $M H$ s were evenly distributed to 25 cells. Each $M H$ randomly selected a moving direction
$[0,360]$ degree and speed $[30,90] \mathrm{km}$. The distribution of speeds and directions of $M H \mathrm{~s}$ is listed in Table 2 . The $w_{1}$ and $w_{2}$ was equal to 0.7 and 0.3 , and the threshold of mobility, $\delta$, was 0.1 . The time interval of updating statuses of $M H \mathrm{~s}, T$, was one second. The transmit power, $\mu$, was 105 units, and the path-loss exponent, $\gamma$, was 30 
Table 1. Multimedia service types.

\begin{tabular}{cccc}
\hline $\begin{array}{c}\text { Service } \\
\text { class }\end{array}$ & $\begin{array}{c}\text { Required } \\
\text { bandwidth }(\mathrm{kb} / \mathrm{s})\end{array}$ & $\begin{array}{c}\text { Connection } \\
\text { duration }(\mathrm{s})\end{array}$ & $\begin{array}{c}\text { Handoff } \\
\text { priority }\end{array}$ \\
\hline Class 1 & 64 & 60 & 1 \\
Class 2 & $64 * 2$ & $60 * 5$ & 4 \\
Class 3 & $64 * 3$ & $60 * 15$ & 8 \\
\hline
\end{tabular}

Table 2. Distribution of speeds and directions of $\mathrm{MHs}$.

\begin{tabular}{cc}
\hline Velocity and direction & The amount of $M H \mathrm{~s}$ \\
\hline Constant, forward & $75 \%$ \\
Constant, backward & $5 \%$ \\
Accelerated 30\%, forward & $5 \%$ \\
Decelerated 30\%, forward & $5 \%$ \\
Accelerated 60\%, forward & $5 \%$ \\
Decelerated 60\%, forward & $5 \%$ \\
\hline
\end{tabular}

units. Finally, the $R S S^{\text {handoff }}$ and $R S S^{\text {issuehandoff }}$ were 2.061 and 1.569 units.

In simulations, we had investigated the performance of DHPA in terms of the call blocking probability (CBP), call dropping probability (CDP), and bandwidth utilization $(\mathrm{BU})$ by comparing with other ordering strategies, first-in-first-out (FIFO), and signal strength for multimedia communication (SSMC), and No Priority (NP) schemes. In the No Priority scheme, new calls and handoff calls had the same priority and were served arbitrarily. There was no queue implemented in this scheme. In FIFO scheme, the available bandwidth was allocated $\mathrm{MHs}$ based on their arriving order. If there were no channels left, new calls were simply blocked and handoff requests were queued in the handoff queue. In the SSMC scheme, a new call was directly blocked without queuing if there was no available bandwidth in it origin cell.

Figure 13 shows the CBP of the NP, FIFO, SSMC, and DHPA ordering schemes with respect to the increase of call arrival rate. The SSMC and FIFO schemes both had higher CBP than the DHPA scheme as they favored providing bandwidth to handoff calls. Without the design of new call queue, new calls were blocked once a cell had no sufficient bandwidth. Similar to the SSMC and FIFO schemes, the DHPA also prioritized serving handoff calls, but it further implemented a new call queue to avoid immediately blocking new calls when a cell was temporary out of available bandwidth. The NP scheme had the lowest CBP because it did not differentiate between new calls and handoff calls. Available bandwidth was equally shared between new and handoffs calls, leading to the lowest CBP of all four schemes.

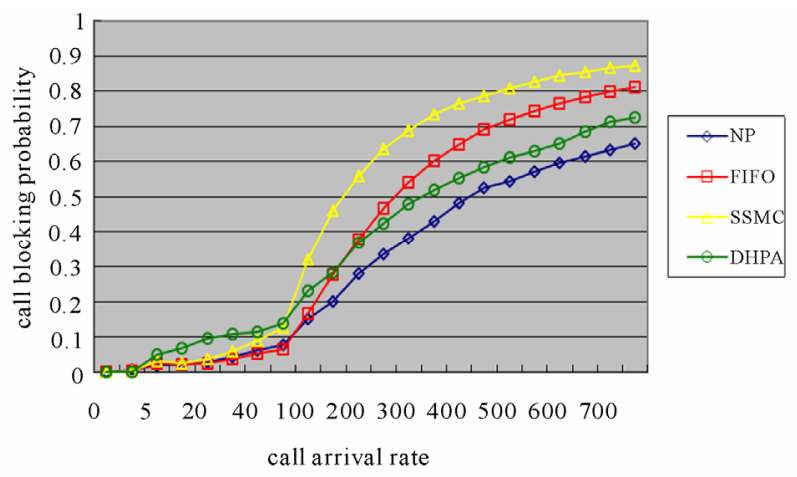

Figure 13. Call blocking probability.

Figure 14 shows the CDP of NP, FIFO, SSMC, and DHPA schemes with the increase of call arrival rate. As shown in the figure, the CDP increases along with the increase of arrival rate for all four schemes. Obviously, the CDP of the proposed DHPA was much lower than that of NP, FIFO, and SSMC on average by $192 \%, 151 \%$ and $100 \%$, respectively. Two key properties revealed the superiority of DHPA in CDP. First, the priority of active handoff queue was adjusted mostly based on the dwelling time which was more suitable to reflect the urgency of handoff, and most importantly, it was updated periodically, unlike the SSMC which statically assigned priority to each queued $M H$. Second, in DHPA scheme bandwidth successfully reserved in target cell by inactive handoff calls which was assumed not to be used for a while was released to save dropping urgent handoff calls. The NP scheme did not provide with handoff queue so that any $M H$ unable to successfully reserve bandwidth in target cell was directly dropped, resulting in high CDP. In the FIFO scheme a handoff priority of a $M H$ was estimated without considering its mobility, and urgent handoff calls might not acquire bandwidth in the target cell for handoff, resulting in a higher CDP than the SSMC and DHPA as well. It was noted that both the DHPA and the SSMC schemes considered SC, RSS, and $\triangle R S S$ in calculating initial handoff priority. However, ignoring urgent handoffing $\mathrm{MHs}$ caused by the change of mobility and the utilization of bandwidth reserved by immobile handoff $M H$ s led to higher CDP in the SSMC than the DHPA.

Figure 15 shows the bandwidth utilization of the NP, FIFO, SSMC, and DHPA schemes with respect to the increase of call arrival rate. The bandwidth utilization of our proposed DHPA obviously was higher than that of FIFO and SSMC on average by $10 \%$ and $9 \%$, and was lower than the NP scheme. With the DHPA scheme, bandwidth successfully reserved by inactive handoff $M H \mathrm{~s}$ was released to urgent handoff $M H \mathrm{~s}$ to avoid service interruption. As a result, the idleness of bandwidth was reduced, thus bandwidth utilization of the DHPA 


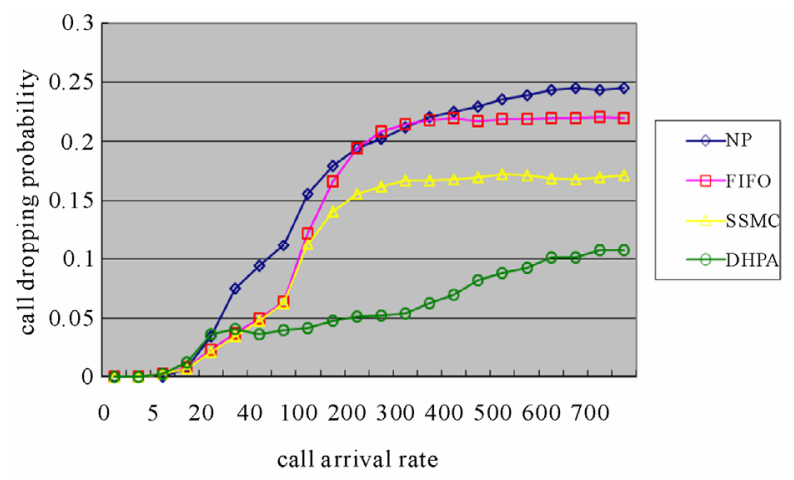

Figure 14. Call dropping probability.

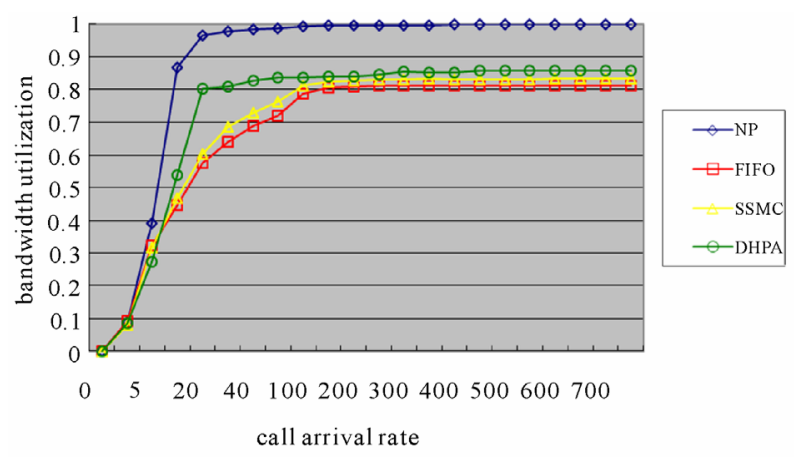

Figure 15. Bandwidth utilization.

was better than that of FIFO and SSMC schemes. As far as the bandwidth utilization was concerned, the NP scheme was the best solution which utilized any available bandwidth to satisfy new and handoff $M H$ s. Without prioritize providing available bandwidth to handoff $M H \mathrm{~s}$, as shown in Figure 14, the CDP of the NP scheme was much higher than that of the DHPA.

\section{Conclusions}

When the target cell cannot provide adequate bandwidth for a handoff call, instead of dropping the request, queuing approach is the major technique to save dropping handoff $M H \mathrm{~s}$. How to effectively and dynamically reflect the urgency of queued handoff $M H \mathrm{~s}$ is very crucial to maintain the QoS of a $M H$. In general, the mobility of a $M H$ in the handoff area is varied with time, where a $M H$ may move toward target cell or backward to origin cell with various speeds changed with time, or even stand still. As a result, the urgency of handoffing to target cell is varied also. It is vital to dynamically adjust the handoff priority of $M H$ s based on their mobility to timely reflect the urgency of handoff in order to ensure QoS for on-going calls without interruption. In this paper, we proposed the DHPA scheme which not only dynamically adjusts handoff priority of $M H \mathrm{~s}$ based on their mobility but also further reallocates idle bandwidth, reserved by inactive $M H \mathrm{~s}$ in immobile set, to urgent handoff $M H \mathrm{~s}$ to reduce the CDP. The simulation results shown that the proposed DHPA scheme could have high BU and much lower CDP with acceptable CBP.

\section{References}

[1] M. El-Kadi, S. Olariu and H. Abdel-Wahab, "A RateBased Borrowing Scheme for QoS Provisioning in Multimedia Wireless Networks," IEEE Transactions on Parallel and Distributed Systems, Vol. 13, No. 2, 2002, pp. 156-166. doi:10.1109/71.983943

[2] B. M. Epstein and M. Schwartz, "Predictive Qos Based Admission Control for Multiclass Traffic in Cellular Wireless Networks," IEEE Journal on Selected Areas in Communications, Vol. 18, No. 3, 2000, pp. 523-534. doi:10.1109/49.840209

[3] A. Mlla, M. El-Kadi, S. Olariu and P. Todorova, "A Fair Resource Allocation Protocol for Multimedia Wireless Networks," IEEE Transactions on Parallel and Distributed Systems, Vol. 14, No. 1, 2003, pp. 63-71. doi:10.1109/TPDS.2003.1167371

[4] F. R. Yu, V. W. S. Wong and V. C. M. Leung, "A New QoS Provisioning Method for Adaptive Multimedia in Wireless Networks," IEEE Transactions on Vehicular Technology, Vol. 57, No. 3, 2008, pp. 1899-1909. doi:10.1109/TVT.2007.907023

[5] O. T. W. Yu and V. C. M. Leung, "Adaptive Resource Allocation for Prioritized Call Admission over an ATM-Based Wireless PCN," IEEE Journal on Selected Areas in Communications, Vol. 15, No. 7, 1997, pp. 1208-1225. doi:10.1109/49.622906

[6] D. K. Kim and D. K. Sung, "Characterization of Soft Handoff in CDMA Systems," IEEE Transactions on Vehicular Technology, Vol. 48, No. 4, 1999, pp. 1195-1202. doi: $10.1109 / 25.775368$

[7] C. C. Chao and W. Chen, "Connection Admission Control for Mobile Multiple-Class Personal Communication Network," IEEE Journal on Selected Areas in Communication, Vol. 15, No. 8, 1997, pp. 1618-1626.

[8] S. Chen and K. Nahrstedt, "Distributed Quality-of-Service Routing in Ad-Hoc Networks," IEEE Journal on Selected Areas in Communication, Vol. 17, No. 8, 1999, pp. 1488-1505. doi:10.1109/49.780354

[9] V. Li and W. J. Liao, "Wireless Multimedia Networks," Proceedings of International Symposium on Parallel Architectures, Algorithms and Networks, Makati City, May 2002, pp. 69-73.

[10] W. I. Kim and C. S. Kang, “An Adaptive Soft Handover Algorithm for Traffic-Load Shedding in the WCDMA Mobile Communication System," IEEE Wireless Communications and Networking, Vol. 2, 2003, pp. 12131217.

[11] H. Matsuoka, M. Yoshimura and T. Ohya, "A Robust Method for Soft IP Handover," IEEE Internet Computing, Vol. 7, No. 2, 2003, pp. 18-24. doi:10.1109/MIC.2003.1189185 
[12] T. Fong, P. Henry, K. Leung, Q. Xiaoxin and N. Shankaranarayanan, "Radio Resource Allocation in Fixed Broadband Wireless Networks," IEEE Transactions on Communications, Vol. 46, No. 6, 1998, pp. 806-818. doi:10.1109/26.681421

[13] M. Chiu and M. Bassiouni, "Predictive Scheme for Handoff Prioritization in Cellular Networks Based on Mobile Positioning," IEEE Journal on Selected Areas in Communication, Vol. 18, No. 3, 2000, pp. 510-522. doi: $10.1109 / 49.840208$

[14] M. El-Kadi, S. Olariu and H. Abdel-Wahab, "A RateBased Borrowing Scheme for QoS Provisioning in Multimedia Wireless Networks," IEEE Transactions on Parallel and Distributed Systems, Vol. 13, No. 2, 2002, pp. 156-166. doi:10.1109/71.983943

[15] B. Epstein and M. Schwartz, "Predictive QoS-Based Admission Control for Multiclass Traffic in Cellular Wireless Networks," IEEE Journal on Selected Areas in Communication, Vol. 18, No. 3, 2000, pp. 523-534. doi:10.1109/49.840209

[16] R. S. Chang and S. J. Leu, "Handoff Ordering Using Signal Strength for Multimedia Communications in Wireless Networks," IEEE Transactions on Wireless Communications, Vol. 3, No. 5, 2004, pp. 1526-1532. doi:10.1109/TWC.2004.833515

[17] O. T. W. Yu and V. C. M. Leung, "Adaptive Resource Allocation for Prioritized Call Admission over an ATMBased Wireless PCN," IEEE Journal on Selected Areas in Communications, Vol. 15, No. 7, 1997, pp. 1208-1225. doi: $10.1109 / 49.622906$

[18] C. Oliverira, J. B. Kim and T. Suda, "An Adaptive Bandwidth Reservation Scheme for High-Speed Multimedia Wireless Networks," IEEE Journal of Selected Areas in Communications, Vol. 16, No. 6, 1998, pp. 858-874. doi:10.1109/49.709449

[19] H. H. Liu, "Guard Channel Sharing Strategies in Integrated Voice/Data Mobile Networks," Advanced Information Networking and Applications, Vol. 2, 2004, pp. 79-82.

[20] R. Qian and G. Feng, "A Minimum-Dwelling-Time Prioritization Scheme for Handoff in Mobile Cellular Systems," International Conference on Communication Technology, Beijing, 22-24 October 1998, p. 5.

[21] S. Tekiny and B. Jabbari, "A Measurement-Based Prioritization Scheme for Handovers in Mobile Cellular Networks," IEEE Journal on Selected Areas in Communications, Vol. 10, No. 8, 1992, pp. 1343-1350. doi: $10.1109 / 49.166761$

[22] H. G. Ebersman and O. K. Tonguz, "Handoff Ordering Using Signal Prediction Priority Queuing in Personal Communication Systems," IEEE Transactions on Vehicular Technology, Vol. 48, No. 1, 1999, pp. 20-35.

[23] C. Choi, M. Kim, T. Kim and S. J. Kim, “Adaptive Bandwidth Reservation Mechanism Using Mobility Probability in Mobile Multimedia Computing Environment," Proceedings of the 25th Annual IEEE Conference on Local Computer Networks, Tampa, 8-10 November 2000, pp. 76-85. doi:10.1109/LCN.2000.891011 